\title{
Efficacy Assessment of Epidural Blockade and Tranexamic Acid Application in idiopathic Scoliosis Surgery
}

\author{
DOI: $10.17691 / \mathrm{stm} 2018.10 .4 .20$
}

Received/23May, 2018

A.A. Ezhevskaya, MD, PhD, Leading Researcher, Anesthesiology and Resuscitation Department ${ }^{1}$;

Zh.B. Prusakova, MD, PhD, Senior Researcher, Anesthesiology and Resuscitation Department';

V.. Zagrekov, MD, PhD, Head of Anesthesiology and Resuscitation Department';

A.V. Sosnin, PhD, Assistant Professor';

M. Milenovic, MD, PhD, Chair, WFSA Education Committee, Faculty of Medicine, University of Belgrade3;

Clinical Center of Serbia, Center for Anesthesiology and Resuscitation, Emergency Center ${ }^{4}$

1Privolzhsky Research Medical University, 10/1 Minin and Pozharsky Square, Nizhny Novgorod, 603005, Russia;

${ }^{2}$ National Research University Higher School of Economics, 25/12 Bolshaya Pecherskaya St., Nizhny Novgorod, 603155, Russia;

${ }^{3}$ Faculty of Medicine, University of Belgrade, 8 Doktora Subotica, Belgrade, 11000, Serbia;

${ }^{4}$ Clinical Center of Serbia, Center for Anesthesiology and Resuscitation, Emergency Center, 2 Pasterova,

Belgrade, 11000, Serbia

The aim of the study was to evaluate the effect of administering tranexamic acid, alone and combined with epidural anesthesia, on postoperative blood loss and donated blood transfusion in patients undergoing corrective scoliosis surgery.

Materials and Methods. 160 patients aged 15-18, were assigned to 4 groups in a double-blinded manner to receive one of the two anesthesia techniques: general anesthesia (sevoflurane and fentanil), or general anesthesia (sevoflurane) with an epidural block (thoracic epidural anesthesia with $0.5 \%$ ropivacaine and fentanil) and one of the two solutions for antifibrinolytic therapy. These solutions were tranexamic acid (group GT and group ET), and 0.9\% saline as placebo (group GP and group EP). Tranexamic acid was administered in groups ET and GT before skin incision - a bolus of $15 \mathrm{mg} / \mathrm{kg}$ followed by IV infusion of $2 \mathrm{mg} / \mathrm{kg} / \mathrm{h}$ up to the end of surgery. For a statistical analysis, we employed non-parametric methods and linear regression.

Results. Group GT demonstrated a statistically significant reduction in the volume of intraoperative blood loss by $28.9 \%$ as compared with group GP. The groups with epidural anesthesia (EP, ET) demonstrated a statistically significant reduction in the volume of intraoperative blood loss by 43.3 and $21.9 \%$, as compared with the groups without the epidural component in anesthesia (GP and GT). The standalone use of tranexamic acid in patients without epidural anesthesia led to a reduction in their intraoperative blood loss by $28.9 \%$. Epidural anesthesia contributed to the limitation of changes in the biochemical data of hemostasis/fibrinolysis system during surgery. At the same time, hypercoagulation changes dominated in group GP. Hence, groups EP and ET did not require hemotransfusion, while group GT featured a much lower need for it. There were no severe complications in all the groups. The effect of fibrinolysis inhibition was complementary.

Conclusion. Tranexamic acid and epidural anesthesia, administered separately or in combination with each other, can be safely and effectively applied to reduce perioperative blood loss and the need for hemotransfusion during corrective surgery for idiopathic scoliosis in adolescents. The combination of the methods produces the biggest blood-saving effect. Still, the standalone use of tranexamic acid in scoliosis surgery is a safe, cheap, and effective method of blood-saving.

Key words: tranexamic acid; epidural anesthesia; blood loss; hemostasis; fibrinolysis; idiopathic scoliosis.

\section{Introduction}

Spinal deformity surgery is one of the most complicated procedures performed in pediatric orthopedics and corrective surgery for adolescent idiopathic scoliosis (AIS) and is regarded as a major surgical procedure. Patients undergoing major spinal surgery are at risk of excessive blood loss, which may result in immunologic reactions, transmission of infections, or even transfusion-related acute lung injury.
There is also the risk of spinal epidural hematoma formation, which may lead to spinal cord or cauda equine compression [1]. In the surgical procedures, allogenic blood transfusion and long operative times have been associated with increased post-surgical infection [2]. Significant operative time $(>6-8 \mathrm{~h})$, blood loss (500-2500 ml), neurologic injury, and cardiovascular complications can occur [3]. Surgical infections in spinal deformity surgery involve a very high morbidity to the patient and the family, along with a high price tag to the

Corresponding author: Anna A. Ezhevskaya, e-mail: annaezhe@yandex.ru 
medical system. Bearing in mind that operative time and transfusions (and hence blood loss) increase patient morbidity, all clinicians should seek ways to minimise these risk factors.

Corrective surgery for scoliosis is a long-continued and highly traumatic procedure accompanied by considerable blood loss and hence causing a set of complicated reactions at the level of neuroendocrinal tension, metabolism intensification and marked shifts in oxygen delivery [4, 5]. Massive blood loss in corrective surgery for scoliosis continues to be a burning issue in anesthesiology and orthopedics. It is well known that the use of donated blood components to compensate for the blood loss is associated with a risk of hemotransmissive infections, post-transfusion reactions and complications, allogenic immunisation, and immunosuppresion [6-8]. The etiology of blood loss in scoliosis surgery is of multifactor nature, the major role being played by the deficiency of coagulation factors, and intensified fibrinolysis potentially resulting in its increase $[9,10]$.

Tranexamic acid is a synthetic antifibrinolytic drug which has the resultant effect of forming a reversible complex with plasminogen and plasmin. It inhibits fibrinolysis, prevents the lysis of fibrin coagulum and acts toward partial blocking of the platelet aggregation induced by plasmin $[11,12]$.

A randomized double-blind trial conducted in corrective surgery for scoliosis in children has revealed that the administration of tranexamic acid in a dose of $10 \mathrm{mg} / \mathrm{h}$ before the skin incision and its constant transfusion during the operation in a dose of $\mathrm{mg} / \mathrm{kg} / \mathrm{h}$ permit reducing blood loss by $28 \%$ as compared with the administration of placebo $(1230 \pm 535$ vs. $1784 \pm 733 \mathrm{ml}$; $p<0.01)$. It also reduces the need to transfuse donated erythrocytes [13]. Given the multi-factor nature of blood loss, no standalone method allows of a complete abandonment of donated blood transfusion in corrective surgery for scoliosis.

The use of antifibrinolytic drugs has been debated for years and antifibrinolytic dosing schedules vary (IV tranexamic acid from medium $\left(\mathrm{mg} \cdot \mathrm{kg}^{-1}\right)$ to very large doses up to $100 \mathrm{mg} \cdot \mathrm{kg}^{-1}$ as a loading dose with 1 or $10 \mathrm{mg} \cdot \mathrm{kg}^{-1} \cdot \mathrm{h}^{-1}$ infusion) [5, 14-22]. Tranexamic acid has been used in different studies to reduce blood losses during spinal surgery. There are no definite protocols or dosages, but it seems that a total dose of $1 \mathrm{~g}$ administered intraoperatively, is sufficient for most adult patients [23-25].

In the case of massive or diffuse bleeding, a point-of-care testing as thromboelastography and thromboelastometry will provide the correct information to direct a goal-directed transfusion therapy [4, 23-28].

Tranexamic acid has been successfully used as part of an enhanced recovery pathway in hip and knee replacement [28], and a recent meta-analysis of spinal surgery studies concludes that the use of tranexamic acid appears to be effective in reducing blood loss, the volume of blood transfusion, the transfusion rate and the postoperative partial thromboplastic time [29-32].

Among other blood-saving methods, controlled hypotension is commonly employed, which aims to maintain the target value of mean arterial pressure (MAP) at $50-60 \mathrm{~mm} \mathrm{Hg}$. It is achieved by using remifentanil, increasing the depth of anesthesia (with volatile anesthetics), and by administering clonidine, labetalol, or sodium nitroprusside. In a recent survey, $26 \%$ of institutions employing this method defined an acceptable MAP (independent of controlled hypotension) within the range of $55-70 \mathrm{~mm} \mathrm{Hg}$. At the same time, 35\% of institutions reported the use of controlled hypotension and acute normovolemic hemodilution. The concurrent use of more than one blood-sparing technique was not addressed by the survey $[5,19]$.

One of the most efficient methods in the way of reducing MAP is the epidural block, which is extensively used in orthopedic surgery of major joints, but not in spinal surgery where its application is limited. However, some researchers regard a MAP of over $85 \mathrm{~mm} \mathrm{Hg}$ as a criterion of exclusion from the study, thus corroborating the fact of its impact on the amount of blood loss.

Such factors as the massive trauma induced, significant operative time, inevitable and considerable blood loss, and original cardiopulmonary dysfunctions account for high surgical and anesthetic risks of spinal surgery. There remains a significant incidence of dangerous postoperative complications and rather a high level of postoperative mortality reaching $1.06 \%$ $[4-6,33]$. A number of studies have proved that epidural anesthesia is one of the most effective, though invasive, methods of intra- and postoperative pain relief. It is successfully employed both in general and orthopedic surgery and ensures the blood-saving effect [29, 34]. Several studies have demonstrated that the underlying disease, surgery, and blood loss cause adverse changes in central and peripheral hemodynamics [35]. As it has been revealed, the basic factors influencing the patient's hemodynamic status in corrective surgery for scoliosis include the intraoperative position, the exposure time, and the intensity of the cardiodepressant and vasodilatory action of drugs used for anesthetic protection. It has been established that the method of inhalation anesthesia on the basis of sevoflurane entails the development of a more unfavorable hydration condition of the lungs as compared with the method of total intravenous anesthesia on the basis of propofol. The former increases the risk of respiratory and cardiopulmonary failure [35].

So far, no uniform opinion has been formed as to the mechanisms according to which regional anesthesia influences the condition of the hemostatic system and the incidence of complications. The potential mechanisms include hemodynamic factors, the systemic action of the local anesthetic, and, finally, limitations to stress-induced hypercoagulation [8]. At the same time, isolated studies have reported the successful application 
of epidural anesthesia in patients with spinal disorders and its positive effect on the hemostatic system [34].

The aim of the research consists in evaluating the effect of administering tranexamic acid, alone and combined with epidural anesthesia, on postoperative blood loss and donated blood transfusion in patients undergoing corrective surgery for scoliosis.

\section{Materials and Methods}

After obtaining the institutional local ethics committee approval and informed written consent, we randomized 160 patients aged 14-18 to four different techniques of anesthesia for surgical deformity correction. The patients had a primary diagnosis of AIS or idiopathiclike scoliosis, and were recruited during the period from Jan. 2015 to Jan. 2017. The patients underwent posterior-only spinal instrumentation and fusions at the Academic Medical Centre of the Privolzhsky Research Medical University by one and the same orthopedic spine surgery team.

The present trial utilises a prospective double-blind cohort design. The exclusion criteria were the following: ASA physical status over III, age under 14 years or over 18 years, body mass index (BMI) more than $30 \mathrm{~kg} / \mathrm{m}^{2}$, allergy to local anesthetics or opioids, current opioid use, active infectious processes, neurological disorders, abnormal coagulation tests, renal or hepatic failure, lack of cooperation, and inability to comprehend or perform verbal and physical assessments.

Further excluded were patients with osteogenesis imperfecta, mean perioperative arterial pressure over $85 \mathrm{~mm} \mathrm{Hg}$, previous spinal surgery or revision surgery, intake of medication contradicting the standardised protocol, neuromuscular scoliosis or infantile/juvenile scoliosis

We retrieved the data on the patients' age, sex, preoperative hemoglobin, height, weight, and BMI from the medical records. Intraoperative data included the number of spinal levels fused, the number of SmithPetersen osteotomies, the duration of surgery (hours), the estimated blood loss, and the number of allogenic blood units transfused.

Upon arrival at the operation room, the patients were assigned (by a computer-generated randomisation) in a double-blinded manner to receive one of the two anesthesia techniques: general anesthesia with sevoflurane and fentanil, or general anesthesia with an epidural block and one of the two solutions for antifibrinolytic therapy. These solutions had been prepared by our pharmacy and were essentially (1) tranexamic acid in a 50-ml syringe (group GT and group ET), and (2) $0.9 \%$ saline as placebo in a $50-\mathrm{ml}$ syringe (group GP and group EP).

All the four groups were equal as to the number of patients.

Tranexamic acid or the placebo solution were administered according to the following plan: $15 \mathrm{~min}$ prior to the skin incision patients received a bolus dose of $15 \mathrm{mg} / \mathrm{kg}$; next, intravenous infusion at a rate of $1 \mathrm{mg} / \mathrm{kg} / \mathrm{h}$ was effected until the wound closure; in the early postoperative period an additional $250 \mathrm{mg}$ was administered.

The induction of anesthesia in both groups was performed with IV propofol $(2 \mathrm{mg} / \mathrm{kg}$ ) and fentanyl (2 $\mathrm{mg} / \mathrm{kg}$ ). Prior to intubation, rocuronium was used to ensure the neuromuscular blockade at a dose of $0.6 \mathrm{mg} / \mathrm{kg}$.

General anesthesia was maintained with sevoflurane and oxygen, with the minimum alveolar anesthetic concentration of $1.2 \%$. A subclavian catheter to monitor the central venous pressure was inserted, along with an indwelling bladder catheter to avoid urinary retention. Before the section and the most traumatic stages of surgery, patients in group GT and group GP underwent general anesthesia with sevoflurane; they were infused with fentanil at a rate of $0.002-0.003 \mathrm{mg} / \mathrm{kg} / \mathrm{h}$. Whenever the blood pressure or heart rate increased by over 20 $30 \%$, the depth of anesthesia was deemed inadequate, and hemodynamic control was ensured with incremental doses of IV fentanyl (50-100 $\mu \mathrm{g})$.

Prior to skin incision, the patients from the epidural anesthesia groups were given a sidelying epidural blockade at two levels (Th4-Th7 and Th12-L2). An epidural bolus dose of $0.15 \mathrm{ml} / \mathrm{kg}$ of $0.5 \%$ ropivacaine $/ 2 \mu \mathrm{g} / \mathrm{ml}$ fentanyl was administered at each level. General anesthesia was maintained with sevoflurane and oxygen, the minimum alveolar anesthetic concentration being 0.7 . The depth of anesthesia was assessed according to the BIS monitor readings (Covidien, USA) within a target range of $50-60 \%$ in both groups.

Hypotension (blood pressure less than $20 \%$ of the baseline measurement) was treated with incremental doses of IV ephedrine ( $5 \mathrm{mg}$ ).

Prior to wound closure, the surgeon positioned two catheters for postoperative pain relief in all the patients at the following levels: Th4-Th7 and Th12-L2. The same surgeon performed all the operations. Sevoflurane administration was stopped at the beginning of skin closure. Patients were awakened and their tracheas were extubated at the conclusion of surgery if they met the standard extubation criteria. At the conclusion of surgery, all the patients were extubated. They were then transferred to the ICU for close monitoring during the next $24 \mathrm{~h}$.

Following the extubation, the patients were evaluated neurologically to detect potential deficits.

Upon the patients' admission to the ICU, a continuous epidural infusion of $0.2 \%$ ropivacaine $/ 2 \mu \mathrm{g} / \mathrm{ml}$ fentanyl at a rate of $0.05 \mathrm{ml} / \mathrm{kg}^{-1} / \mathrm{h}$ was started in each epidural catheter.

All the patients received standard perioperative monitoring. All the patients received paracetamol $15 \mathrm{mg} / \mathrm{kg}$ IV every $6 \mathrm{~h}$ and ondansetron 2-4 mg IV every $4 \mathrm{~h}$ if nausea or vomiting were present. Upon 
admission to the ICU, the patients were asked to assess their pain, and if the VAS score was over $40 \mathrm{~mm}$ at rest, titration with IV morphine administered by a nurse was performed. IV patient-controlled analgesia (PCA) was then started with a PCA device programmed to deliver morphine in bolus doses of $2 \mathrm{mg}$, with a lockout duration of $8 \mathrm{~min}$ and an unrestricted total dose.

The primary end point of this study was to determine the estimated blood volume. It was calculated by adding together the blood volume collected both by the suction and cell-saver system and with the surgical sponges, which were weighed. The total irrigation fluid was measured strictly by the nurses prior to use in the operative field.

During surgery, autoblood samples were taken from all the patients to be processed in C.A.T.S. cellsaving apparatus (Fresenius, Germany) and to return washed erythroconcentrate with a RBC plasma ratio of $75-80 \%$. Infusion therapy was performed according to the blood loss rate and to maintain a CVP of $5-8 \mathrm{~mm} \mathrm{Hg}$ by colloids and crystalloids. Fresh frozen plasma or packed red blood cells were transfused if blood loss was $25-30 \%$ or over $30 \%$ of the circulating blood volume respectively, the red blood parameters being strictly controlled. Systemic hemodynamics (systolic blood pressure, diastolic blood pressure, MAP, systemic vascular resistance index) was monitored with NICCOMO (Medis, Germany) and NIHON COHDEN (Japan) apparatuses, and with capnography devices (VAMOS; Drager, Germany). Coagulogram was taken twice postoperatively; complete blood count was performed at various stages of the surgery and after it. Blood loss, autohemotransfusion, infusion therapy, and the volumes of the donated media transfused were measured during surgery and on postoperative day 1 (POD1).

Our null hypothesis was that no differences would exist between the groups with regard to administering tranexamic acid or performing epidural blockade. All the continuous data are presented as mean and standard deviation. The assumption of normality was checked using the Kolmogorov-Smirnov test. Statistical analysis was conducted according to the type of data distribution and the conditions of criterion applicability with the aid of STATISTICA software package (version 10.0, StatSoft Inc.). We employed nonparametric statistical methods and linear regression.

Values were reported as the mean and the standard deviation for parametric data, or the median with a $25-75 \%$ interquartile range for nonparametric data. Parametric variables were checked for normality using the Shapiro-Wilk test. The Mann-Whitney $U$ statistical test was applied to nonparametric and ordinal variables. Comparison of the

Table 1 two groups was performed using the Student $t$ test (for normally distributed data) and the Wilcoxon signed-rank test. Differences were considered statistically significant at $p<0.05$.

\section{Results}

In all the patients undergoing dorsal spondylodesis, the surgery employed the transpedicular fixation technique and multilevel spinal vertebrotomies (osteotomies). The all groups have had no significant difference in duration of surgery, sex, age, starting hemoglobin, BMI, or maximum preoperative Cobb between the groups being found (Table 1).

The present study was conducted to establish correlations existing between perioperative blood loss and the type of anesthesia, between the blood loss and the application of fibrinolysis inhibitors, tranexamic acid in particular. In the course of the trial, group GP demonstrated a statistically significant reduction in the volume of intraoperative blood loss by $28.8 \%$ as compared with group GT (Table 2), while the volume of postoperative blood loss stayed the same $(p=0.10)$. The trial has revealed a moderate direct correlation between the duration of surgery and blood loss in all the patient groups, irrespective of the type of anesthesia chosen or the blood-saving method employed. Therefore, it was essential that the operative time parameter should not affect the results (Figure 1). The groups did not differ with respect to the duration of surgery, or to the number of fusion segments.

The groups of patients who were administered epidural anesthesia during surgery (EP, ET) demonstrated a statistically significant reduction in the volume of intraoperative blood loss as compared with the groups without the epidural component in anesthesia (GP and GT) - 43.3 and $21.9 \%$, respectively (see Table 2). From the statistical standpoint, postoperative blood loss was significantly lower in groups EP and ET as compared with the other ones, which did not display any significant differences.

A comparative assessment of the number of donated blood components transfused in the groups has

Clinical and demographic data (mean $\pm S D$ )

\begin{tabular}{lcccc}
\hline \multicolumn{1}{c}{ Parameter } & $\begin{array}{c}\text { Group GT } \\
(\mathrm{n}=40)\end{array}$ & $\begin{array}{c}\text { Group GP } \\
(\mathrm{n}=40)\end{array}$ & $\begin{array}{c}\text { Group ET } \\
(\mathrm{n}=40)\end{array}$ & $\begin{array}{c}\text { Group EP } \\
(\mathrm{n}=40)\end{array}$ \\
\hline Sex (F/M) & $73 / 3$ & $38 / 2$ & $35 / 5$ & $38 / 2$ \\
\hline Age (years) & $15.6 \pm 1.2$ & $16.1 \pm 0.6$ & $15.2 \pm 1.1$ & $15.9 \pm 0.7$ \\
\hline Preoperative $\mathrm{Hb}(\mathrm{g} / \mathrm{dl})$ & $13.2 \pm 0.8$ & $12.8 \pm 0.6$ & $13.0 \pm 1.1$ & $12.9 \pm 0.9$ \\
\hline BMl & $16.8 \pm 0.8$ & $17.2 \pm 1.2$ & $17.3 \pm 0.9$ & $16.9 \pm 1.1$ \\
\hline Preoperative Cobb angle (degrees) & $64.3 \pm 6.8$ & $64.5 \pm 7.3$ & $63.7 \pm 6.2$ & $64.6 \pm 8.1$ \\
\hline Postoperative Cobb angle (degrees) & $17.9 \pm 0.9$ & $17.7 \pm 1.1$ & $18.2 \pm 0.6$ & $17.8 \pm 0.8$ \\
\hline ASA score & $\mathrm{I}$ & $\mathrm{I}$ & $\mathrm{I}$ & $\mathrm{I}$ \\
\hline
\end{tabular}


Table 2

Intraoperative and postoperative data (mean \pm SD)

\begin{tabular}{lcccc}
\hline \multicolumn{1}{|c}{ Parameter } & $\begin{array}{c}\text { Group GT } \\
(\mathrm{n}=40)\end{array}$ & $\begin{array}{c}\text { Group GP } \\
(\mathrm{n}=40)\end{array}$ & $\begin{array}{c}\text { Group ET } \\
(\mathrm{n}=40)\end{array}$ & $\begin{array}{c}\text { Group EP } \\
(\mathrm{n}=40)\end{array}$ \\
\hline Intraoperative blood loss $(\mathrm{ml})$ & $734.1 \pm 28.5^{*}$ & $1050.2 \pm 65.7$ & $380.8 \pm 40.2^{*}$ & $430.6 \pm 45.7^{*}$ \\
\hline Intraoperative blood loss $(\%)$ & $17.3 \pm 1.2^{*}$ & $27.2 \pm 1.6$ & $9.8 .3 \pm 1.3^{*}$ & $11.4 \pm 0.9^{*}$ \\
\hline Postoperative blood loss $(\mathrm{ml})$ & $120.9 \pm 8.5$ & $180.6 \pm 10.3$ & $75.6 \pm 7.5^{*}$ & $82.2 \pm 9.9^{*}$ \\
\hline Blood transfusion $(\mathrm{ml})$ & $276.5 \pm 34.2^{*}$ & $565.8 \pm 23.7$ & None & None \\
\hline Mean arterial pressure $(\mathrm{mm} \mathrm{Hg})$ & $69.5 \pm 6.3^{* \#}$ & $78.7 \pm 4.7^{* \#}$ & $57.6 \pm 5.7^{* \#}$ & $58.5 \pm 4.9^{* \#}$ \\
\hline Length of surgery (min) & $220.5 \pm 12.7$ & $226.3 \pm 11.5$ & $215.7 \pm 13.7$ & $222.6 \pm 10.8$ \\
\hline Number of levels fused & $12.5 \pm 0.8$ & $12.8 \pm 1.0$ & $13.1 \pm 7.8$ & $12.7 \pm 8.6$ \\
\hline POD3 Hb (g/dl) & $91.2 \pm 3.7^{* \#}$ & $7.9 \pm 0.2^{* \#}$ & $11.5 \pm 2.4^{* \#}$ & $10.3 \pm 0.9^{* \#}$ \\
\hline
\end{tabular}

* $p<0.05$ - statistically significant differences between the groups;

\# $p<0.05$ - as compared to the initial value.

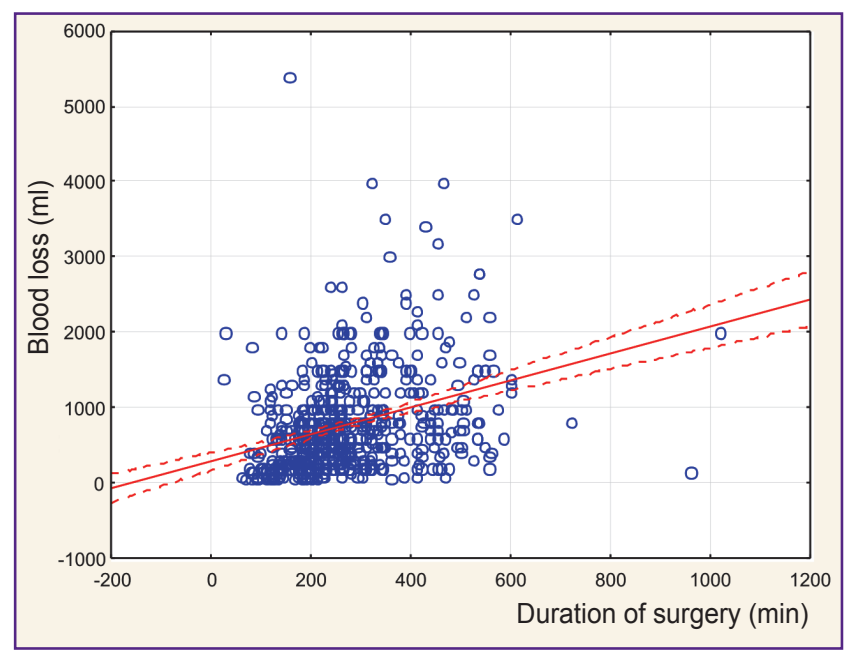

Figure 1. Moderate correlation between the duration of surgery and blood loss in all groups (Spearmen's rankorder correlation: $r=0.40 ; p=0.0000 ; 95 \%$ conf. int.)

revealed that most hemo- and plasma transfusions were performed in group GP, the volume of media transfused being significantly greater statistically. In groups GT, $E P$, and ET transfusions of packed donated red blood cells were not performed on POD1, while groups EP and $\mathrm{ET}$ did not require hemotransfusion throughout the perioperative period.

In all the patient groups, the progressive changes in hemoglobin, hematocrit, and erythrocytes were unidirectional, both during surgery and postoperatively. Ten percent of group GT patients were transfused packed red blood cells on POD3 due to anemia (with the hemoglobin indicators below $7.5 \mathrm{~g} / \mathrm{dl}$ ). An examination of changes in the hemoglobin level has revealed its strong direct correlation with perioperative blood loss $(r=0.78 ; p=0.0001)$. Groups EP and ET featured stable hemoglobin levels, to a minimum of $9.0 \mathrm{~g} / \mathrm{dl}$ (see Table 2). Group GP demonstrated the lowest levels on POD3 $(7.9 \pm 0.2 \mathrm{~g} / \mathrm{dl})$, which necessitated hemotransfusion in $10 \%$ of patients. Thus, the most stable red blood cell lineage was observed in the group which received a combined anesthesia with sevoflurane, epidural anesthesia, and tranexamic acid.

Hemotransfusion-related complications were only observed in two patients from group GP in the form of shiver and nettle rash, when fresh frozen plasma was being transfused. The process was immediately stopped and the reactions were swiftly arrested by administering glucocorticosteroids and antihistamines.

A complex analysis of the hemostatic system was conducted in all the patients preoperatively, on POD1, and POD3. In the early postoperative period, all the groups displayed the preponderance of hypercoagulation shifts characterised by a reduction (within the normal range) in the activated partial thromboplastin time (A-PTT) and by an increase in the concentration of fibrinogen and soluble fibrin complexes (SFC). On POD3 also showed the indications of moderate fibrin formation in group GP, which was indicated by a fairly high level of thrombinemia markers and the markers of fibrinolysis activation (soluble fibrin and D-dimer) (Table 3).

The fact of intravascular fibrin formation was further corroborated by the observed reduction in the level of plasminogen and an increase in the rate of the tissue-type plasminogen activator, which features a high affinity to intravascular fibrin deposits. However,

Table 3

\section{Basic indicators of the hemostatic system}

\begin{tabular}{|c|c|c|c|c|}
\hline \multirow{2}{*}{$\begin{array}{l}\text { Time points } \\
\text { (stages) }\end{array}$} & \multirow{2}{*}{ Groups } & \multicolumn{3}{|c|}{ Parameters } \\
\hline & & A-PTT & SFC & Xlla-DF \\
\hline \multirow{4}{*}{ Preoperative } & GT & $37.9 \pm 8.2$ & $40.7 \pm 7.4$ & $7.1 \pm 0.4$ \\
\hline & GP & $36.5 \pm 5.7$ & $40.3 \pm 4.8$ & $6.8 \pm 0.3$ \\
\hline & ET & $38.7 \pm 7.6$ & $37.5 \pm 3.8$ & $6.4 \pm 0.2$ \\
\hline & EP & $38.9 \pm 3.9$ & $40.4 \pm 5.8$ & $7.8 \pm 0.7$ \\
\hline \multirow{4}{*}{ POD1 } & GT & $28.5 \pm 1.8^{\#}$ & $37.6 \pm 2.9$ & $13.6 \pm 0.5^{\star \#}$ \\
\hline & GP & $26.4 \pm 5.1$ & $60.8 \pm 5.9^{* \#}$ & $15.5 \pm 0.8^{* \#}$ \\
\hline & ET & $33.4 \pm 2.6^{\#}$ & $36.4 \pm 3.7$ & $9.5 \pm 0.3$ \\
\hline & EP & $32.6 \pm 2.8$ & $48.6 \pm 6.8$ & $8.9 \pm 0.2$ \\
\hline \multirow{4}{*}{ POD3 } & GT & $32.4 \pm 4.7^{\text {*\# }}$ & $97.3 \pm 3.9^{* \#}$ & $20.9 \pm 0.2^{* \#}$ \\
\hline & GP & $31.3 \pm 3.7^{\text {*\# }}$ & $120.7 \pm 9.6^{* \#}$ & $22.9 \pm 1.5^{\text {*\# }}$ \\
\hline & ET & $40.3 \pm 5.5^{\star}$ & $58.5 \pm 6.5^{\text {*\# }}$ & $13.8 \pm 0.7^{\text {*\# }}$ \\
\hline & EP & $38.5 \pm 3.8$ & $60.5 \pm 8.4^{* \#}$ & $15.3 \pm 0.7^{* \#}$ \\
\hline
\end{tabular}

${ }^{*} p<0.05$ - statistically significant differences between the groups; ${ }^{*} p<0.05$ — as compared to the initial value; A-PTT activated partial thromboplastin time; SFC - soluble fibrin complexes; XIla-DF — XIla-dependent fibrinolysis. 
no group revealed any significant consumption of physiological anticoagulants - antithrombin III and protein $\mathrm{C}$. The mean values stayed within the reference value range in all the groups. It was only by POD3 that the thrombinemia and the fibrinolysis activation markers had returned to the initial values in all the groups, with low molecular weight heparin being administered for prophylactic purposes. By POD3, however, groups GP and GT had revealed a more statistically significant inhibition of fibrinolysis - a longer time of XIladependent fibrinolysis by 27 and $20 \%$, respectively. This attested to a higher risk of blood clot organisation and thromboembolic events in the groups mentioned, while in the groups with epidural anesthesia this indicator did not rise above $12 \%$ (see Table 3 ).

No event of deep vein thrombosis or pulmonary embolism was detected during the patients' hospital stay and the study conducted; nor did we not encounter any bleeding event, which would require surgical reintervention.

The present trial has revealed the statistically significant moderate correlations between intraoperative (and, to a lesser extent, postoperative) blood loss and fibrinolysis activity at POD1 in all the patient groups (Figure 2, see Table 3). These correlations are of multidirectional nature. Furthermore, we have uncovered unidirectional and statistically significant changes in blood loss and antithrombin III, which testify to the absence of significant consumption of physiological anticoagulants. On POD1 we observed the indications of moderate but clinically apparent thrombinemia and of fibrinolysis activation, which manifested themselves in an increased amount of fibrin and fibrinogen degradation products, SFC in particular. The changes in the latter were unidirectional with the blood loss $(p=0.001)$.

The analysis of systemic hemodynamics has revealed more stable indices of MAP in patients from the groups with sevoflurane-maintained anesthesia.

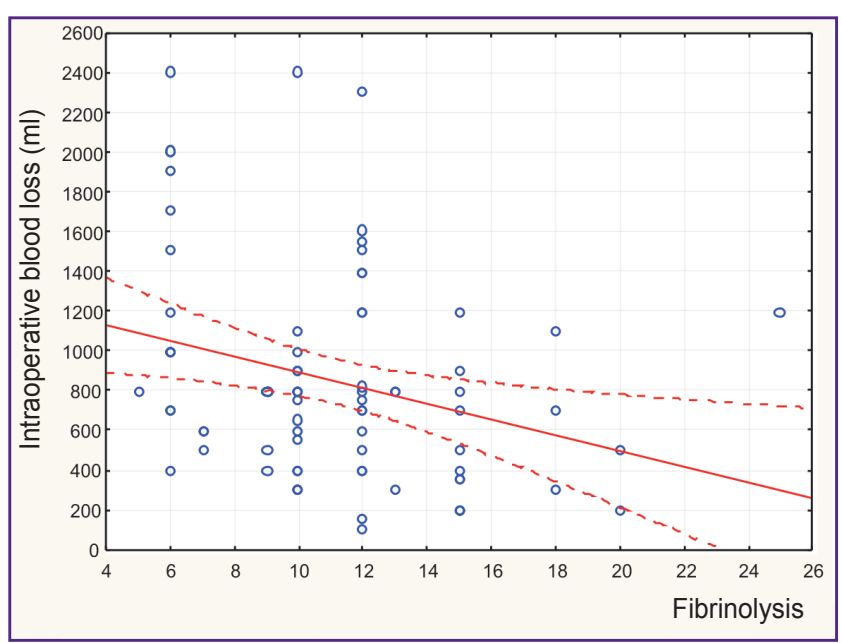

Figure 2. Moderate correlation between blood loss and fibrinolysis at POD1 in all groups $(r=-0.41 ; p=0.001 ; 95 \%$ conf. int.)
Systemic hemodynamics was sustained at the level of moderate hypotension. Patients from the groups with epidural anesthesia exhibited the heart rate, cardiac output, and MAP indices which were 10-15\% lower than in patients from groups GP and GT. It is statistically significant and was due to sympaticolysis at the level of the segments blocked (see Table 2). Patients who received epidural anesthesia developed hypotension in $15 \%$ of cases, which necessitated the administration of vasoconstrictors. Patients from group GP displayed a statistically significant increase in systolic blood pressure, MAP, and cardiac output by $15-20 \%$ at the most traumatic stages - spinal skeletisation, screw positioning, and the deformity correction point, which partly accounts for major volumes of blood loss and hemotransfusion.

\section{Discussions}

The trial conducted has indicated that a major bloodsaving effect of up to $40-50 \%$ of the loss is markedly pronounced when the epidural blockade is effected as a component of general anesthesia in corrective surgery for scoliosis. Furthermore, the standalone use of tranexamic acid as a part of the anesthesia plan in patients without epidural anesthesia has led to a reduction in their intraoperative blood loss by $28.8 \%$, which confirms the data of previous research [11, 13, $22,36,37]$. Our study has also shown that the groups receiving tranexamic acid featured a lower percentage of perioperative blood loss in relation to the volume of blood circulation, which corroborates the results of another retrospective study which used the same index.

At the same time, the epidural blockade bloodsaving effect which has been achieved is most likely to be related to such hemodynamic factor of regional anesthesia as the redistribution of blood circulation, especially in the system of capacitance vessels and namely in the inferior vena cava system, which is directly connected to the system of epidural veins and the venous system of the spongy bone tissue in the spine.

Of particular interest, however, is the fact that tranexamic acid administration according to the plan described and in the context of epidural anesthesia has not led to any significant changes in intra- and postoperative blood loss $(p=0.12)$. Therefore, the effects of a combined usage of the techniques are not merely summed up arithmetically. A major reduction in perioperational blood loss during this type of surgery is most likely to be due to the effects of sympaticolysis in epidural anesthesia, to controlled hypotension, hypotonia in epidural veins, and intraosseous pressure. In these conditions, the effect of fibrinolysis inhibition is complementary, affecting the volume of perioperative blood loss and hemotransfusion requirements only insignificantly.

Nevertheless, given the complicated and multifactor nature of blood loss, no particular method can fully 
eliminate the necessity of transfusing donated blood components in major spinal surgery.

We cannot rule out the fact that for a more definite conclusion, a continuation of the study might be required, with a greater number of patients in each group.

Following the previous research, we also believe that maintaining the MAP in the groups is a major factor determining the hemorrhage rate [3-5, 20-22].

Surgical hemostasis is an important factor not to be bypassed. To avoid the situation when the technical factor might influence the results, we made a point of making the groups maximally homogeneous, with one and the same team performing surgery, using the same instruments, and following the uniform hemostatic technique - coagulation, hemostatic sponge, specific procedures. Medical literature has very few instances where this factor is excluded [3, 22].

The data obtained indicate that the reaction of the hemostatic system to a major spinal surgery consists in the simultaneous increase in coagulation and fibrinolysis. It appears that the role of thoracic epidural anesthesia is to limit the activation of the hemostasis/fibrinolysis system.

In spinal surgery, the condition of the hemostasis/ fibrinolysis system in the intraoperative period is characterised by relative hypocoagulation proceeding from the infusion load, and by dilutional coagulopathy accompanied by fibrinolysis activation, which results in yet greater blood loss. Postoperatively, however, stable hypercoagulation replaces this condition in all the patient groups, including those receiving epidural anesthesia. Yet, it is epidural anesthesia that limits the excessive activation of the hemostasis/fibrinolysis system, which is essential to prevent both thromboembolic and hemorrhagic complications postoperatively. Changes in the hemostatic system primarily depend on the blood loss and the related volume of infusion therapy, hemodilution, protein deficiency, anemia, dilutional coagulopathy, consumption coagulopathy, etc.

All the patient groups have displayed statistically significant multidirectional and weak correlations between blood loss and the changes in the number of red blood cells and platelets. Furthermore, no critical thrombocytopenia or hemorrhage has been observed in any of the groups. The aggregatogram data stayed within the normal range in all the groups at all the stages of the trial.

The nature of changes in the hemostatic system can largely depend on the type of surgery. As distinct from orthopedic operations causing hypercoagulation and inhibiting fibrinolysis, abdominal surgery can enhance fibrinolysis, which results in greater blood loss and which has proved to be typical of spinal surgery. Certain types of orthopedic operations, e.g. those on major joints (lasting under $4 \mathrm{~h}$, which is not exceeding long) feature a statistically significant inverse correlation between the operative time and the blood loss rate [26]. On the contrary, major spinal interventions are characterised by a direct rather than inverse correlation of this kind the longer the operation lasts, the faster the blood loss rate grows, especially after $4-5 \mathrm{~h}$ of surgery. In addition, this correlation is moderate $(r=0.56 ; p=0.001)$, which is apparently explained by the growing dilutional coagulopathy due to the prolonged volume-expanding infusion therapy.

Our study has proved that tranexamic acid can be safely administered to patients, as no one of them has developed any side-effects related to its use, either local or systemic. Other similar studies corroborate the safety of tranexamic acid $[21,26]$, with the number of complications being negligible or comparable with placebo. Furthermore, no complications related to the epidural component of anesthesia have been registered.

Thus, tranexamic acid boasts a powerful potential of its own for reducing blood loss to $30 \%$ and lessening the need for allogenic blood transfusions in the absence of the epidural component of anesthesia. Tranexamic acid also serves as an additional medical drug inhibiting enhanced fibrinolysis in the context of epidural anesthesia/analgesia.

\section{Conclusions}

Tranexamic acid and epidural anesthesia administered separately or in combination with each other can be safely and effectively applied to reduce perioperative blood loss and hemotransfusion requirements during corrective surgery for idiopathic scoliosis in adolescents. The combination of the methods produces the biggest blood-saving effect Still, the standalone use of tranexamic acid is a safe, cheap, and effective method of blood-saving. To support the conclusions drawn, further blind, prospective and randomized research is required.

Acknowledgment. The authors would like to thank Marina Presnyakova, MD PhD, Department of Biochemistry, and Ekaterina Donchenko, Head of Innovation Department and Technology Transfer, Privolzhsky Research Medical University, Nizhny Novgorod, Russia, for their outstanding efforts with the laboratory analysis and data collection, and for their extraordinary technical support.

Financial support. No funds were received in support of this work. The manuscript submitted does not contain information about medical device(s)/drug(s). No benefits in any form have been or will be received from a commercial party related directly or indirectly to the subject of this manuscript.

The device(s)/drug(s) is/are FDA approved or approved by corresponding national agency for this indication. No funds were received in support of this work. Relevant financial activities outside the submitted work: consultancy, royalties, and payment for lectures.

Conflict of interest. The authors declare that they have nothing to disclose. 


\section{References}

1. Zhang F., Wang K., Li F.N., Huang X., Li Q., Chen Z., Tang Y.B., Shen H.X., Song Q.X. Effectiveness of tranexamic acid in reducing blood loss in spinal surgery: a meta-analysis. BMC Musculoskelet Disord 2014; 15: 448, https://doi. org/10.1186/1471-2474-15-448.

2. Halanski M.A., Elfman C.M., Cassidy J.A., Hassan N.E., Sund S.A., Noonan K.J. Comparing results of posterior spine fusion in patients with AIS: are two surgeons better than one? J Orthop 2013; 10(2): 54-58, https://doi.org/10.1016/j. jor.2013.03.001.

3. Fisahn C., Jeyamohan S., Norvell D.C., Tubbs R.S., Moisi M., Chapman J.R., Page J., Oskouian R.J. Association between allogeneic blood transfusion and postoperative infection in major spine surgery. Clin Spine Surg 2017; 30(7): E988-E992, https://doi.org/10.1097/bsd.0000000000000539.

4. Lamperti M., Tufegdzic B., Avitsian R. Management of complex spine surgery. Curr Opin Anaesthesiol 2017; 30(5): 551-556, https://doi.org/10.1097/aco.0000000000000494.

5. Palmer G.M., Pirakalathanan P., Skinner A.V. A multicentre multi-national survey of anaesthetists regarding the range of anaesthetic and surgical practices for paediatric scoliosis surgery. Anaesth Intensive Care 2010; 38(6): 1077-1084.

6. Fowler A.J., Ahmad T., Phull M.K., Allard S., Gillies M.A., Pearse R.M. Meta-analysis of the association between preoperative anaemia and mortality after surgery. Br J Surg 2015; 102(11): 1314-1324, https://doi.org/10.1002/ bjs. 9861 .

7. Berney M.J., Dawson P.H., Phillips M., Lui D.F., Connolly P. Eliminating the use of allogeneic blood products in adolescent idiopathic scoliosis surgery. Eur $J$ Orthop Surg Traumatol 2015; 25(Suppl 1): S219-S223, https://doi. org/10.1007/s00590-015-1624-3.

8. Tzortzopoulou A., Cepeda M.S., Schumann R., Carr D.B. Antifibrinolytic agents for reducing blood loss in scoliosis surgery in children. Cochrane Database Syst Rev 2008; 3: CD006883, https://doi.org/10.1002/14651858.cd006883.

9. Chandra S., Kulkarni H., Westphal M. The bloody mess of red blood cell transfusion Critical Care 2017; 21(Suppl 3): 310, https://doi.org/10.1186/s13054-017-1912-x.

10. Bosch P., Kenkre T.S., Londino J.A., Cassara A., Yang C., Waters J.H. Coagulation profile of patients with adolescent idiopathic scoliosis undergoing posterior spinal fusion. J Bone Joint Surg Am 2016; 98(20): e88, https://doi. org/10.2106/jbjs.16.00114.

11. Sethna N.F., Zurakowski D., Brustowicz R.M., Bacsik J., Sullivan L.J., Shapiro F. Tranexamic acid reduces intraoperative blood lossin pediatric patients undergoing scoliosis surgery. Anesthesiology 2005; 102(4): 727-732, https://doi.org/10.1097/00000542-200504000-00006.

12. Neilipovitz D.T. Tranexamic acid for major spinal surgery. Eur Spine J 2004; Suppl 1: S62-S65, https://doi. org/10.1007/3-540-27394-8_10.

13. Neilipovitz D.T., Murto K., Hall L., Barrowman N.J., Splinter W.M. A randomized trial of tranexamic acid to reduce blood transfusion for scoliosis surgery. Anesth Analg 2001; 93(1): 82-87, https://doi.org/10.1097/00000539-200107000-00018.

14. Xu C., Wu A., Yue Y. Which is more effective in adolescent idiopathic scoliosis surgery: batroxobin, tranexamic acidor a combination? Arch Orthop Trauma Surg 2012; 132(1): 25-31, https://doi.org/10.1007/s00402-011-1390-6.
15. Johnson D.J., Johnson C.C., Goobie S.M., Nami N., Wetzler J.A., Sponseller P.D., Frank S.M. High-dose vs lowdose tranexamic acid to reduce transfusion requirements in pediatric scoliosis surgery. J Pediatr Orthop 2017; 37(8): e552e557, https://doi.org/10.1097/bpo.0000000000000820.

16. McNicol E.D., Tzortzopoulou A., Schumann R., Carr D.B., Kalra A. Antifibrinolytic agents for reducing blood loss in scoliosis surgery in children. Cochrane Database Syst Rev 2016; 9: CD006883, https://doi.org/10.1002/14651858. cd006883.pub3.

17. Lykissas M.G., Crawford A.H., Chan G., Aronson L.A., Al-Sayyad M.J. The effect of tranexamic acid in blood loss and transfusion volume in adolescent idiopathic scoliosis surgery: a single-surgeon experience. J Child Orthop 2013; 7(3): 245249, https://doi.org/10.1007/s11832-013-0486-7.

18. Faraoni D., Goobie S.M. The efficacy of antifibrinolytic drugs in children undergoing noncardiac surgery: a systematic review of the literature. Anesth Analg 2014; 118(3): 628-636, https://doi.org/10.1213/ane.0000000000000080.

19. Verma K., Lonner B., Dean L., Vecchione D., Lafage V. Reduction of mean arterial pressure at incision reduces operative blood loss in adolescent idiopathic scoliosis. Spine Deformity 2013; 1(2): 115-122, https://doi.org/10.1016/j. jspd.2013.01.001.

20. Verma K., Errico T., Diefenbach C., Hoelscher C., Peters A., Dryer J., Huncke T., Boenigk K., Lonner B.S. The relative efficacy of antifibrinolytics in adolescent idiopathic scoliosis: a prospective randomized trial. J Bone Joint Surg Am 2014; 96(10): e80, https://doi.org/10.2106/jbjs.I.00008.

21. Wahlquist S., Wongworawat M., Nelson S. When does intraoperative blood loss occur during pediatric scoliosis correction? Spine Deform 2017; 5(6): 387-391, https://doi. org/10.1016/j.jspd.2017.04.004.

22. Sui W.Y., Ye F., Yang J.L. Efficacy of tranexamic acid in reducing allogeneic blood products in adolescent idiopathic scoliosis surgery. BMC Musculoskelet Disord 2016; 17: 187, https://doi.org/10.1186/s12891-016-1006-y.

23. Mehra T., Seifert B., Bravo-Reiter S., Wanner G., Dutkowski P., Holubec T., Moos R.M., Volbracht J., Manz M.G., Spahn D.R. Implementation of a patient blood management monitoring and feedback program significantly reduces transfusions and costs. Transfusion 2015; 55(12): 2807-2815, https://doi.org/10.1111/trf.13260.

24. Yagi M., Hasegawa J., Nagoshi N., lizuka S., Kaneko S., Fukuda K., Takemitsu M., Shioda M., Machida M. Does the intra-operative tranexamic acid decreaseoperative blood loss during posterior spinal fusion fortreatment of adolescent idiopathic scoliosis? Spine 2012; 37(21): E1336E1342, https://doi.org/10.1097/brs.0b013e318266b6e5.

25. Yuan C., Zhang H., He S. Efficacy and safety of using antifibrinolytic agents in spine surgery: a meta-analysis. PLOS One 2013; 8(11): e82063, https://doi.org/10.1371/journal. pone.0082063.

26. Ker K., Edwards P., Perel P., Shakur H., Roberts I. Effect of tranexamic acid on surgical bleeding: systematic review and cumulative meta-analysis. BMJ 2012; 344: e3054, https://doi.org/10.1136/bmj.e3054.

27. Theusinger O.M., Levy J.H. Point of care devices for assessing bleeding and coagulation in the trauma patient. Anesthesiol Clin 2013; 31(1): 55-65, https://doi.org/10.1016/j. anclin.2012.10.006

28. Lee Y.C., Park S.J., Kim J.S., Cho C.H. Effect of tranexamic acid on reducing postoperative blood 
loss in combined hypotensive epidural anesthesia and general anesthesia for total hip replacement. J Clin Anesth 2013; 25(5): 393-398, https://doi.org/10.1016/j. jclinane.2013.02.006.

29. Wang M., Zheng X.F., Jiang L.S. Efficacy and safety of antifibrinolytic agents in reducing perioperative blood loss and transfusion requirements in scoliosis surgery: a systematic review and meta-analysis. PLoS One 2015; 10(9): e0137886, https://doi.org/10.1371/journal.pone.0137886.

30. Irwin A., Khan S.K., Jameson S.S., Tate R.C. Copeland C., Reed M.R. Oral versus intravenous tranexamic acid in enhanced-recovery primary total hip and knee replacement: results of 3000 procedures. Bone Joint $J$ 2013; 95-B(11): 1556-1561, https://doi.org/10.1302/0301$620 x .95 b 11.31055$.

31. Stowers M.D., Lemanu D.P., Coleman B., Hill A.G. Munro J.T. Review article: perioperative care in enhanced recovery for total hip and knee arthroplasty. J Orthop Surg (Hong Kong) 2014; 22(3): 383-392, https://doi.org/10.1177/23 0949901402200324.

32. Wainwright T.W., Immins T., Middleton R.G. Enhanced recovery after surgery (ERAS) and its applicability for major spine surgery. Best Pract Res Clin Anaesthesiol 2016; 30(1): 91-102, https://doi.org/10.1016/j.bpa.2015.11.001.

33. Ljungqvist O., Scott M., Fearon K.C. Enhanced recovery after surgery: a review. JAMA Surg 2017; 152(3): 292-298, https://doi.org/10.1001/jamasurg.2016.4952.

34. Wang T.L., Qi Y.Q., Yang B.X., Zhao L. Epidural anesthesia can protect fibrinolytic function after surgery. Beijing Da Xue Xue Bao Yi Xue Ban 2004; 36(4): 383-389.

35. Brown Z.E., Görges M., Cooke E., Malherbe S., Dumont G.A., Ansermino J.M. Changes in cardiac index and blood pressure on positioning children prone for scoliosis surgery. Anesthesia 2013; 68(7): 742-746, https://doi. org/10.1111/anae.12310.

36. da Rocha V.M., de Barros A.G., Naves C.D., Gomes N.L., Lobo J.C., Villela Schettino L.C., da Silva L.E. Use of tranexamic acid for controlling bleeding in thoracolumbar scoliosis surgery with posterior instrumentation. Rev Bras Ortop 2015; 50(2): 226-231, https://doi.org/10.1016/j.rboe.2015.03.007.

37. Ng B.K., Chau W.W., Hung A.L., Hui A.C., Lam T.P., Cheng J.C. Use of tranexamic acid (TXA) on reducing blood loss during scoliosis surgery in Chinese adolescents. Scoliosis 2015; 10: 28, https://doi.org/10.1186/s13013-015-0052-9. 